Grant/research support from: Medimmune, Vanessa L. Bryant Grant/research support from: CSL

DOI: 10.1136/annrheumdis-2020-eular.3507

\section{AB0145 1 EFFECTS OF IMMUNOSUPPRESSIVE MEDICATION ON TYPE I INTERFERON ACTIVATION: IN VITRO ANALYSIS SHOWS A DOWNREGULATING EFFECT ON IFN ACTIVATION OF HYDROXYCHLOROQUINE AND ASPIRIN}

M. J. Wahadat ${ }^{1,2}$, M. Lourens ${ }^{1}$, E. Huijser ${ }^{1}$, C. G. Van Helden-Meeuwsen ${ }^{1}$, S. Kamphuis ${ }^{2}$, M. Versnel ${ }^{1}{ }^{1}$ Erasmus MC, Dept. Immunology, Rotterdam, Netherlands; ' Sophia Children's Hospital, Erasmus MC, Dept. Pediatric Rheumatology, Rotterdam, Netherlands

Background: Systemic Lupus Erythematosus (SLE) is prototypic Interferon (IFN) driven autoimmune disease characterized by an increased expression of type-I IFN stimulated genes, known as the IFN signature. The inhibitory effects of various drugs like Hydroxychloroquine and more recently Aspirin on IFN inducing pathways $(1,2)$ led to the idea that some standard of care drugs might decrease the IFN score in patients. Data on the in vitro effect of immunosuppressive medication on IFN activation are limited. Testing immunosuppressive agents for their effect on IFN activation in vitro will give insight into the mechanisms of IFN activation in vivo and the effect of immunosuppressive medication on this activation.

Objectives: To study the effect of immunosuppressive medication on the type-I IFN signature in an in vitro model

Methods: Freshly isolated human PBMCs were stimulated for 24 hours with or without CpG-A or Imiquimod (IQ) or transfected with the cGAS agonist G3-YSD to induce IFN upregulation through the TLR7/9- and DNA Sensing Receptor-pathway respectively. To assess the direct role of the medication on the downstream pathway of the IFNAR PBMCs were stimulated with IFN-a2b. Aspirin, diclofenac, $\mathrm{HCQ}$, Mycophenolate Mofetil (MMF) and prednisone were added separately to these cultures followed by analysis of MxA by qPCR as a readout for IFN type I activation. Cell viability in all culture conditions was above $85 \%$.

Results: The type I IFN activation induced by CpG-A, IQ, G3-YSD and IFN$\mathrm{a} 2 \mathrm{~b}$ was significantly reduced after addition of Aspirin. Addition of diclofenac showed a trend towards reduced levels in all conditions. HCQ was able to significantly reduce the TLR7/9 induced IFN activation by CpG-A and IQ while MMF and prednisone did not show an effect in any of the culture conditions.

Conclusion: The IFN activation induced by the stimulation of various IFN inducing pathways was significantly reduced by Aspirin and HCQ in an in vitro model. Combining both clinical and in vitro data from our longitudinal cohort of childhood-onset SLE patients will elucidate the effect of different immunosuppressive drugs on the type-I IFN signature in these patients.

References:

[1] Kuznik A, Bencina M, Svajger U, Jeras M, Rozman B, Jerala R. Mechanism of endosomal TLR inhibition by antimalarial drugs and imidazoquinolines. J Immunol. 2011;186(8):4794-804.

[2] Dai J, Huang YJ, He X, Zhao M, Wang X, Liu ZS, et al. Acetylation Blocks cGAS Activity and Inhibits Self-DNA-Induced Autoimmunity. Cell. 2019;176(6):1447-60 e14.

Disclosure of Interests: None declared

DOI: 10.1136/annrheumdis-2020-eular.5631

\section{AB0146 \\ DRUG DEPENDENT ALTERATIONS IN B-CELL REPERTOIRE IN SYSTEMIC LUPUS ERYTHEMATOSUS PATIENTS WITH LOW DISEASE ACTIVITY}

S. Zenz ${ }^{1}$, B. Dreo2, B. Prietl ${ }^{3,4}$, S. Kofler ${ }^{3,4}$, H. Sourij ${ }^{3,4}$, A. Lackner ${ }^{1}$, M. D'orazio ${ }^{1}$, K. Brickmann ${ }^{1}$, J. Gretler ${ }^{1}$, F. Fürst-Moazedi ${ }^{1}$, M. Stradner ${ }^{1}$, W. Graninger ${ }^{1}$, H. P. Brezinsek ${ }^{1}{ }^{1}$ Medical University of Graz, Internal Medicine, Division of Rheumatology and Immunology, Graz, Austria; ${ }^{1}$ Medical University of Graz, Internal Medicine, Division of Rheumatology and Immunology, Graz, Austria; ${ }^{3}$ Medical University of Graz, Internal Medicine, Division of Endocrinology and Diabetology, Graz, Austria; ${ }^{4} \mathrm{CBmed} \mathrm{GmbH}$ - Center for Biomarker Research in Medicine, Graz, Austria

Background: B-cells play a major role in the pathogenesis and perpetuation of the immune response in systemic lupus erythematosus (SLE). So far, B-cell subtypes have been studied well, but the precise mechanisms of the B-cell alterations during disease activity and during remission, depending on different medication, are still unclear.

Objectives: The aim of our study was to investigate the drug dependent alterations in the B-cell repertoire of SLE patients with low disease activity (SLEDAI $-2 \mathrm{~K} \leq 4)$.
Methods: Peripheral blood samples from 39 patients suffering from SLE (mean \pm SD; age $43 \pm 13$ years, $87.2 \%$ females, disease duration $11.1 \pm 7$ years) were drawn over 2 years. All SLE patients were in remission or low disease activity (median \pm SE, SLEDAI of $2 \cdot 0 \pm 1.5$ ). B-cells were characterized using CD19, CD20, CD5, CD27 antibodies and were grouped in naïve $\left(\lg D^{+} 27^{-}\right)$ non-switched memory (IgD $\left.{ }^{+}, \mathrm{CD} 27^{+}\right)$, memory (IgD- CD27 $\left.7^{+}\right), \mathrm{B} 1\left(\mathrm{CD}^{+} 27^{-}\right)$ and $\mathrm{MBL}$-like $\left(\mathrm{CD}^{++}\right) \mathrm{B}$-cells. A quantitative flow cytometric bead-based assay (QuantiBRITE PE kit from Becton Dickinson) was used for the estimation of CD19 antibodies bound per cell. Further, CD38 and CD86 antibodies were used to characterize the B-cell subsets. All cytometric measurements were performed using a standardized BD LSR Fortessa platform. After 3 years of follow-up, patients' data about disease activity and current medication were obtained.

Results: 22 SLE patients were treated with hydroxychloroquine (85.8\%) and 19 patients received mycophenolate mofetil (MMF; $n=14 ; 54.6 \%$ ) or azathioprine (AZA; $n=5 ; 19.5 \%) .5$ patients were treated with other DMARDs. Independently of hydroxychloroquine and/or MMF, no significant differences were seen in naïve, non-switched memory, post-switched memory, plasma blasts, B1- or MBL-like B-cells. Patients treated with AZA had significantly lower naïve B-cells (mean \pm SD, 39.3 $\pm 6.7 v s$. $73.1 \pm 19.3 \% ; p=0.028$ ), but had significantly higher IgD-post switched B-cells $(31.2 \pm 9.1$ vs. $12.5 \pm 9.2 \%$; $p=0.028$, respectively) compared with no AZA-treatment. Interestingly, activated B-cells (5.5 \pm 1.5 vs $1.8 \pm 1.1 \% ; p=0.009$ ) were significantly higher in AZA-treated. After 3 years of follow-up, almost all patients were in remission (median \pm SE, SLEDAI of 2.0 \pm 2.0 ), except of 3 patients with a SLEDAI of $\geq 6$. Interestingly, those patients had at baseline, statistically higher naïve B-cells $(p=0.041)$ and lower B1-like B-cells $(p=0.020)$ compared with patients with low disease activity.

Conclusion: Our results suggest that independently of hydroxychloroquine and/or MMF treatment, all patients with low disease activity had similar normal B-cell subsets. Interestingly, in the small group of patients who were treated with $A Z A$, a reduced regeneration of $B$-cells was shown. Patients with higher disease and high naïve B-cells showed an increased disease activity after three years.

Acknowledgments: The research was performed in "CBmed" and funded by the Austrian Federal Government within the COMET K1 Centre Program, Land Steiermark and Land Wien.

Disclosure of Interests: None declared

DOI: 10.1136/annrheumdis-2020-eular.4412

\section{AB0147 GENE EXPRESSION PROFILES OF PRIMARY SJÖGREN'S SYNDROME ASSOCIATED THROMBOCYTOPENIA IN B-LYMPHOCYTE USING HIGH-THROUGHPUT SEQUENCING}

S. Zhang ${ }^{1}$, J. Qu ${ }^{1}$, L. Wang ${ }^{1}$, M. Li ${ }^{1}$, X. Zeng ${ }^{1} .{ }^{1}$ Peking Union Medical College Hospital, Peking Union Medical College and Chinese Academy of Medical Sciences, Rheumatology, Beijing, China

Background: Primary Sjögren's syndrome(pSS) is a classical systemic autoimmune disease. Thrombocytopenia is one of the hematological manifestations of pSS with great challenges in clinic.

Objectives: To identify the candidate genes and functionally enriched pathways in the immune genesis and progression of primary Sjögren's syndrome (pSS) associated thrombocytopenia.

Methods: High-throughput sequencing was performed on 3 patients with pSS 3 patients with pSS associated thrombocytopenia and 3 healthy individuals. The differentially expressed genes (DEGs) were identified, and function enrichment analyses were processed. The protein-protein interaction network (PPI) was constructed, followed by calculation of topological characteristics and sub-module analysis in order to obtain hub DEGs. The expression of some hub genes was verified by Real-Time PCR in 24 pSS patients.

Results: A total of 19 DEGs were identified. The enriched functions and pathway of the DEGs include Toll-like receptor signaling pathway, Salmonella infection, Viral protein interaction with cytokine and cytokine receptor, NF-kappa B signaling pathway and Human cytomegalovirus infection. Seven hub genes (TNF, IL1B, CXCL8, CCL3, CCL4, CCL3L1, CCL4L1) were identified and pathway enrichment analysis revealed that these genes were mainly enriched in toll-like receptor pathway. The relative expression of the CXCL8 mRNA in B-lymphocytes in patients with pSS associated thrombocytopenia was higher than that in the pSS without thrombocytopenia group. No differences were observed in the IL-1 $\beta$ or TNFa expression between these two groups.

Conclusion: PSS associated thrombocytopenia might be a subset characterized by a systemic inflammatory state. The identification of upregulated genes involved in thrombocytopenia of pSS provides insight in disease pathogenesis and opens avenues for the design of novel therapeutic strategies.

References:

[1] Fox RI: Sjögren's syndrome. Lancet, 2005; 366: 321-31. 